\title{
Adesão a medicamentos de pessoas idosas com hipertensão arterial e fatores
}

\section{relacionados}

\author{
Adhesion to medicines of elderly people with arterial hypertension and related factors \\ Adhesión a medicamentos de personas mayores con hipertensión arterial y factores afines
}

Recebido: 13/09/2021 | Revisado: 17/09/2021 | Aceito: 21/09/2021 | Publicado: 23/09/2021

Juliana Piveta de Lima

ORCID: https://orcid.org/0000-0002-2703-9189 Universidade Federal do Rio Grande, Brasil

E-mail: julianapivettal@hotmail.com

Daiane Porto Gautério Abreu

ORCID: https://orcid.org/0000-0002-1125-4693 Universidade Federal do Rio Grande, Brasil

E-mail: daianeporto@ furg.br

Eliel de Oliveira Bandeira

ORCID: https://orcid.org/0000-0003-1038-1612 Universidade Federal do Rio Grande, Brasil E-mail: bandeira.eliel@hotmail.com

Jamila Geri Tomaschewski Barlem

ORCID: https://orcid.org/0000-0001-9125-9103

Universidade Federal do Rio Grande, Brasil E-mail: jamilabarlem@ furg.br

Luciano Garcia Lourenção

ORCID: https://orcid.org/0000-0002-1240-4702

Universidade Federal do Rio Grande, Brasil E-mail: lucianogarcia@ furg.br

\begin{abstract}
Resumo
Objetivo: Avaliar a adesão a medicamentos de pessoas idosas com hipertensão arterial e seus fatores relacionados. Metodologia: Trata-se de um estudo quantitativo transversal com abordagem exploratório-descritiva. Esta pesquisa faz parte do macroprojeto "Relação entre Letramento Funcional em Saúde, adesão à medicação e funcionalidade em pessoas idosas na estratégia saúde da família", que foi realizado em 10 unidades, totalizando 17 equipes da Estratégia de Saúde da Família, que formam a zona oeste do município de Rio Grande-RS. Resultados: Participaram do estudo 264 pessoas idosas com hipertensão arterial, sendo 36,7\% aderentes e 63,3\% não aderentes. As variáveis que obtiveram relação estatisticamente significativa com as médias de adesão à medicação foram ter diabetes, fazer uso de medicamentos para o sistema digestivo/metabolismo e adquirir a medicação pelo Sistema Único de Saúde ou através de doação. Conclusão: O conhecimento das variáveis que estão relacionadas a adesão à medicação em pacientes idosos com hipertensão arterial contribui para o direcionamento das intervenções e das ações realizadas coordenadamente entre profissionais de saúde, pesquisadores, gestores e formuladores de políticas, a fim de melhor o acesso e a adesão aos tratamentos, minimizando, consequentemente, agravos decorrentes dessa doença crônica.
\end{abstract}

Palavras-chave: Idoso; Adesão à medicação; Hipertensão; Estratégia saúde da família; Enfermagem.

\begin{abstract}
Objective: Assess medication adherence of elderly people with hypertension and its related factors. Methodology: This is a cross-sectional quantitative study with an exploratory-descriptive approach. This research is part of the macro-project "Relationship between Functional Health Literacy, medication adherence and functionality in elderly people in the family health strategy", which was carried out in 10 units, totaling 17 teams of the Family Health Strategy, which form the zone west of the city of Rio Grande-RS. Results: 264 elderly people with arterial hypertension participated in the study, 36.7\% adherent and $63.3 \%$ non-adherent. The variables that had a statistically significant relationship with the mean medication adherence were having diabetes, taking medication for the digestive system/metabolism, and purchasing medication through the Unified Health System or through donation. Conclusion: Knowledge of the variables related to medication adherence in elderly patients with arterial hypertension contributes to directing interventions and actions carried out in coordination between health professionals, researchers, managers and policy makers, in order to improve access and adherence to treatments, thus minimizing harm resulting from this chronic disease.
\end{abstract}

Keywords: Older adult; Adherence to medication; Hypertension; Family health strategy; Nursing. 


\begin{abstract}
Resumen
Objetivo: Evaluar la adherencia a la medicación de los ancianos con hipertensión y sus factores relacionados. Metodología: Se trata de un estudio cuantitativo transversal con abordaje exploratorio-descriptivo. Esta investigación se enmarca dentro del macroproyecto "Relación entre Alfabetización en Salud Funcional, adherencia a la medicación y funcionalidad en ancianos en la estrategia de salud de la familia", el cual se llevó a cabo en 10 unidades, totalizando 17 equipos de la Estrategia de Salud de la Familia, que conforman el zona oeste de la ciudad de Rio Grande-RS. Resultados: participaron del estudio 264 ancianos con hipertensión arterial, 36,7\% adherentes y 63,3\% no adherentes. Las variables que tuvieron una relación estadísticamente significativa con la adherencia media a la medicación fueron tener diabetes, tomar medicación para el sistema digestivo / metabolismo y comprar medicación a través del Sistema Único de Salud o mediante donación. Conclusión: El conocimiento de las variables relacionadas con la adherencia a la medicación en pacientes ancianos con hipertensión arterial contribuye a orientar las intervenciones y acciones que se realizan en coordinación entre profesionales de la salud, investigadores, gestores y formuladores de políticas, con el fin de mejorar el acceso y adherencia a los tratamientos, minimizando así el daño. resultante de esta enfermedad crónica.
\end{abstract}

Palabras clave: Anciano; Adhesión a la medicación; Hipertensión; Estrategia de salud familiar; Enfermería.

\title{
1. Introdução
}

Atrelado ao aumento da população idosa está o crescimento relativo das condições crônicas, em especial as Doenças Crônicas não Transmissíveis (DCNT), que afetam em maior prevalência este segmento da população e são responsáveis por elevados índices de óbitos (Moreira et al, 2013). Nesse sentido, no ano de 2005, cerca de 35 milhões de pessoas morreram por DCNT no mundo (Worl Health Organization, 2005), enquanto que no Brasil, 72,4\% das mortes ocorridas em 2009, foram por estas doenças (DUNCAN et al, 2012). Estes dados demonstram que, embora haja políticas públicas de saúde vigentes, estas não conseguem minimizar os agravos e óbitos decorrentes das doenças crônicas mais comuns nas pessoas idosas (Barreto; Carreira; Marcon, 2015).

As DCNTs mais comuns entre as pessoas idosas são Hipertensão Arterial Sistêmica (HAS) e o Diabetes mellitus (DM). Estas doenças, juntas, são consideradas como os principais fatores de risco para o desenvolvimento de complicações renais, cardíacas e acidente vascular encefálico, resultando, portanto, em altos custos médicos e socioeconômicos. No entanto, a HAS é a DCNT mais predominante entre os idosos e sua prevalência aumenta progressivamente com o envelhecimento (Sociedade Brasileira de Cardiologia, 2016). Além disso, A HAS é considerada como importante fator de risco tanto para o declínio da função cognitiva, quanto da qualidade de vida do idoso e está entre os maiores problemas de saúde pública (Bezerra et al, 2018).

Os tratamentos para doenças crônicas como a HA apresentam características que levam a barreiras específicas para a adesão. Por serem, muitas vezes, assintomáticas e possuírem um regime terapêutico bastante complexo (polifarmácia, várias administrações diárias, dificuldades associadas à via de administração) e longo, o paciente pode ter dificuldades no uso regular dos medicamentos, seja pela ausência de sintomas visíveis ou pela falta de compreensão sobre o curso da doença (Tavares et al, 2016).

As taxas de adesão ao tratamento são baixas. Nos países desenvolvidos apenas $50 \%$ dos pacientes com doenças crônicas aderem ao tratamento, portanto supõe-se que em países em desenvolvimento como o Brasil, essa taxa seja ainda menor devido à carência de recursos e às dificuldades no acesso aos cuidados de saúde (Organização Mundial da Saúde, 2003).

A adesão ao tratamento pode ser compreendida como a extensão do comportamento do indivíduo ao fazer uso de medicamentos, ter cuidados com a alimentação, praticar atividade física, comparecer às consultas médicas e às orientações da equipe de saúde (Abreu; Portela, 2015). Porém, diversos fatores podem interferir nesta prática tais como, dificuldades financeiras, esquema terapêutico, diversos medicamentos prescritos, efeitos adversos, dificuldades no acesso ao sistema de saúde, inadequada relação médico-paciente, a caraterística assintomática da doença e sua cronicidade (Girotto et al, 2013). 
Tendo em vista os índices bastante baixos de adesão à medicamentos de pacientes idosos com hipertensão e os prejuízos para a saúde que o não seguimento adequado do tratamento pode acarretar, torna-se importante conhecer os fatores relacionados a esse comportamento para o planejamento de intervenções adequadas. Nesse sentido, tem-se como questão de pesquisa quais os fatores relacionados à adesão de medicamentos de pessoas idosas e como objetivo avaliar a adesão a medicamentos de pessoas idosas com hipertensão arterial e seus fatores relacionados.

\section{Metodologia}

Trata-se de um estudo quantitativo transversal com abordagem exploratório-descritiva (Gil, 2017). Esta pesquisa faz parte do macroprojeto "Relação entre Letramento Funcional em Saúde, adesão à medicação e funcionalidade em pessoas idosas na estratégia saúde da família", que foi realizado em 10 unidades, totalizando 17 equipes da Estratégia de Saúde da Família (ESF), que formam a zona oeste do município de Rio Grande-RS. Essa zona foi escolhida por ter 100\% de cobertura da ESF, cujo foco de atendimento é a promoção da saúde e a prevenção de doenças, justificando, assim, a extrema importância em conhecer o LFS.

A população selecionada para o estudo foi formada por pessoas idosas adscritas nas 17 equipes da ESF. Foram obedecidos os seguintes critérios de inclusão dos participantes no macroprojeto: ter idade igual ou superior a 60 anos; ser adscrito à área de abrangência da Unidade de Saúde da Família; referir diagnóstico médico de HAS; possuir pelo menos um ano de escolaridade autorreferida como recomenda estudos de avaliação do LFS (Santos; Portella, 2016; Cathery-Goulart et al, 2009; Baker et al, 1999).

Os critérios de exclusão do macroprojeto foram: não conseguir ler o Cartão de Jaeger em nível 20/40, considerado o normal para uma visão periférica com ou sem lentes corretoras ou óculos; não ouvir o sussurro dos lados direito e esquerdo do canal auricular pelo teste do sussurro (Teste de Whisper) e pontuação inferior ao ponto de corte no Mini-Exame do Estado Mental (MEEM). As notas de corte no MEEM foram: 1 a 3 anos de escolaridade $\geq 23$ pontos; 4 a 7 anos de escolaridade $\geq 24$ pontos; $<7$ anos de escolaridade $\geq 28$ pontos (BRASIL, 2007). Os instrumentos Cartão de Jaeger, Teste de Whisper e MiniExame do Estado Mental foram utilizados como recomendam estudos de avaliação do LFS (Santos; Portella, 2016; CatheryGoulart et al, 2009; Baker et al, 1999).

A estimativa da população do município, no ano de 2017, foi de 208 mil habitantes (Brasil, 2010). O cálculo da população de idosos estimada para a área de abrangência do estudo foi realizado tendo por base o percentual deste segmento populacional na cidade de Rio Grande/RS, que, de acordo com o último censo demográfico realizado, é de 13,89\% (Brasil, 2010). A partir da população das 17 equipes incluídas na pesquisa (68.000), foi calculada a porcentagem de idosos (13,89\%), obtendo-se aproximadamente 9.445 pessoas idosas. Destas, se calculou o percentual de pessoas idosas hipertensas com base em dados do VIGITEL 2017, referentes à 2016, que indicou que 60,9\% dos adultos com 65 anos ou mais referiram o diagnóstico de HA em pesquisa telefônica. Assim, chegou-se a estimativa de população do estudo de 5.752 pessoas idosas com HA (Brasil, 2018).

Utilizou-se a fórmula de cálculo de amostras para população finita, com a seguinte fórmula (Miott, 2011):

$$
\mathbf{n}=\left(\mathbf{Z}^{2} \times \mathbf{P} \times \mathbf{Q} \times \mathbf{N}\right) /\left(\mathbf{e}^{2} \times(\mathbf{N}-1)+\mathbf{Z}^{2} \times \mathbf{P} \times \mathbf{Q}\right)
$$

Onde:

Z - nível de significância do estudo = 95\% $(1,96)$;

$\mathrm{P}-$ prevalência do evento = utilizou-se 50\%, que maximiza o tamanho da amostra quando não se sabe a prevalência;

$\mathrm{Q}$ - complementar da prevalência (1-P);

$\mathrm{N}-$ população total $=$ pessoas idosas estimadas na área de abrangência das 17 equipes da ESF; 
$\mathrm{E}-$ erro amostral $=6 \%$.

Aplicando-se a fórmula com os parâmetros descritos anteriormente, obteve-se $\mathrm{n}=254$.

A seleção dos participantes foi por equipe: dividiu-se o n pelas 17 equipes participantes $(254 / 17=14,9)$, obtendo-se 15 pessoas idosas por equipe. Esse número foi ainda dividido pelo número de microáreas de cada equipe. As pessoas idosas alfabetizadas de cada microárea foram selecionadas a partir do cadastro dos Agentes Comunitários de Saúde (ACS), que forneceram uma lista com nome e endereço das mesmas. A partir das listas, foram realizadas visitas domiciliares aos $\mathrm{n}$ de idosos estipulado para cada microárea. Conforme era preenchido o número previamente estipulado para aquela microárea, as coletas eram finalizadas naquele local. Foi realizada visita domiciliar para coleta de dados a 264 pessoas idosas, pois em alguns domicílios residiam mais de uma pessoa idosa.

A coleta de dados foi realizada por meio da aplicação de um questionário de caracterização sociodemográfica e de saúde, e a aplicação de dois instrumentos. O instrumento que verificou o grau de adesão à medicação foi uma versão em português (Oliveira-Filho et al, 2012) da Morisky Medication Adherence Scale (MMAS-8), que mede o comportamento específico de 76 tomar medicamentos (Morisk et al, 2008). A pontuação dessa escala varia de zero a oito, e o grau de adesão foi mensurado de acordo com a seguinte pontuação: alta adesão (oito pontos), média adesão ( 6 a < 8 pontos) e baixa adesão (< 6 pontos), sendo considerados aderentes somente os que obtiveram a pontuação máxima ( 8 pontos), e não aderentes os que pontuaram abaixo desse valor (Oliveira-Filho et al, 2012).

Foi realizado um teste piloto com cinco pessoas idosas fora da área de cobertura da ESF. O tempo médio de aplicação dos instrumentos foi de 20 minutos e não houve necessidade de alterações nos instrumentos, não sendo utilizados os dados obtidos.

A coleta de dados ocorreu no período de julho a dezembro de 2017, pelos mestrandos e bolsistas de iniciação científica envolvidos no macroprojeto que foram capacitados em junho de 2017.

Foram explicados os objetivos, os riscos e os benefícios, e, após aceitarem participar, os idosos assinaram ou colocaram a impressão digital no Termo de Consentimento Livre e Esclarecido (TCLE), em duas vias.

As pessoas idosas foram identificadas nas equipes da ESF a partir dos cadastros dos ACS, que indicaram os participantes de cada microárea que eram alfabetizados e conduziram a equipe de coleta até as residências dos mesmos. Seguiu-se a lista dos ACS, se a pessoa idosa não estava na residência, ou não tinha interesse em participar da pesquisa, realizava-se a visita ao próximo constante na lista. As listas eram organizadas por endereços.

Para análise quantitativa, foi elaborado um banco de dados por meio da digitalização dos resultados no software Statistical Package for the Social Sciences (SPSS) versão 20.0. Foram realizadas: uma análise estatística descritiva, com descrição da frequência absoluta e da frequência relativa para variáveis categóricas, e uso das medidas de tendência central (média) e medidas de dispersão (desvio padrão) para variáveis numéricas; uma análise estatística inferencial, em que foi utilizado o teste ANOVA para verificar a diferença de média da variável LFS para as variáveis categóricas. Foi utilizado o nível de significância de $\mathrm{p}<0,05$.

As variáveis utilizadas no estudo foram: características sociodemográficas (sexo, estado marital, escolaridade, exercício de atividade remunerada, cor e renda); presença de diabetes; medicamentos utilizados para os diferentes sistemas (sistema cardiovascular, sistema digestivo/metabolismo, sistema sanguíneo/hematopoiético, sistema nervoso e sistema respiratório); e a forma com que adquire suas medicações (SUS, farmácia, farmácia popular e doação).

Foram respeitados os preceitos éticos de pesquisa com seres humanos, conforme Resolução 510/2016. O projeto deste estudo foi aprovado pelo Comitê de Ética em Pesquisa (CEP) da Universidade Federal do Rio Grande. 


\section{Resultados}

Participaram do estudo 264 pessoas idosas. A maior parte desses participantes era do sexo feminino, residia com companheiro, possuía mais de quatro anos de estudo, não exercia atividade remunerada, era de cor branca e com renda menor que um salário mínimo (Tabela 1).

Tabela 1 - Distribuição de pessoas idosas com hipertensão arterial segundo características sociodemográficas, Rio Grande do Sul, Brasil, 2017.

\begin{tabular}{ll}
\hline Características sociodemográficas & $\mathbf{N}(\%)$ \\
\hline Sexo & $188(71)$ \\
Feminino & $76(29)$ \\
Masculino & \\
Estado marital & $123(47)$ \\
Sem companheiro & $141(53)$ \\
Com companheiro & \\
Escolaridade & $125(47)$ \\
1 a 4 anos de estudo & $139(53)$ \\
Mais de 4 anos de estudo & \\
Atividade & $64(24)$ \\
Exerce atividade remunerada & $200(76)$ \\
Não exerce atividade remunerada & \\
Cor & $172(65)$ \\
Branco & $92(35)$ \\
Não branco & \\
Renda $*$ & $96(36)$ \\
$\geq 1$ salário & $167(63)$ \\
<1 salário & $1(1)$ \\
Não respondeu
\end{tabular}

* Salário mínimo: R \$ 937,00. Fonte: Autores.

A pontuação mínima da escala de adesão à medicamentos foi zero e a pontuação máxima foi oito pontos, sendo a média de adesão 6,52 com desvio padrão de 1,57. Do total de participantes, 36,7\% são aderentes e 63,3\% não aderentes, sendo 23,1\% com baixa adesão, 40,2 com média adesão e 36,7\% com alta adesão.

Àquelas pessoas idosas que relataram ter diabetes obtiveram maiores médias de adesão à medicação do que as que relataram não ter essa doença (tabela 2), sendo esse resultado estatisticamente significativo ( $\mathrm{p}=0,045$ ). Ainda, houve uma diferença estatisticamente significativa nas médias de adesão à medicação daquelas pessoas idosas que fazem uso de medicamentos para o sistema digestivo/metabolismo $(\mathrm{p}=0,033)$, de maneira que aqueles que fazem uso desse medicamento possuem uma menor adesão à medicação do que aqueles que não fazem uso (Tabela 2). 
Tabela 2 - Distribuição das pessoas idosas com hipertensão arterial segundo presença de diabetes, uso de medicamentos para os diferentes sistemas e média de Adesão à medicação, Rio Grande do Sul, Brasil, 2017.

\begin{tabular}{|c|c|c|c|c|}
\hline Variáveis & $\mathrm{n}(\%)$ & $\begin{array}{l}\text { Média de Adesão } \\
\text { à medicação e } \\
\text { desvio } \\
\text { padrão }(\bar{X} \pm \text { DP })\end{array}$ & Valor $F / t$ & Valor $\mathrm{p}^{*}$ \\
\hline Diabetes & & & 2,012 & $0,045 * *$ \\
\hline Sim & $89(34)$ & $6,63 \pm 1,24$ & & \\
\hline Não & $175(66)$ & $6,46 \pm 1,71$ & & \\
\hline $\begin{array}{l}\text { Medicamento sistema } \\
\text { cardiovascular }\end{array}$ & & & 0,467 & 0,879 \\
\hline Sim & $263(100)$ & $6,52 \pm 1,57$ & & \\
\hline Não & 1 & & & \\
\hline $\begin{array}{l}\text { Medicamento sistema } \\
\text { digestivo/metabolismo }\end{array}$ & & & 2,134 & $0,033 * *$ \\
\hline Sim & $154(58)$ & $6,49 \pm 1,45$ & & \\
\hline Não & $110(42)$ & $6,56 \pm 1,72$ & & \\
\hline $\begin{array}{l}\text { Medicamento sistema } \\
\text { sanguíneo/hematopoiético }\end{array}$ & & & 0,556 & 0,814 \\
\hline Sim & $111(42)$ & $6,59 \pm 1,52$ & & \\
\hline Não & $153(58)$ & $6,47 \pm 1,60$ & & \\
\hline Medicamento sistema nervoso & & & 0,990 & 0,444 \\
\hline Sim & $107(41)$ & $6,50 \pm 1,45$ & & \\
\hline Não & $157(59)$ & $6,53 \pm 1,64$ & & \\
\hline $\begin{array}{l}\text { Medicamento sistema } \\
\text { respiratório }\end{array}$ & & & 0,239 & 0,983 \\
\hline $\operatorname{Sim}$ & $18(7)$ & $6,83 \pm 1,38$ & & \\
\hline Não & $246(93)$ & $6,50 \pm 1,58$ & & \\
\hline
\end{tabular}

* Teste ANOVA. **Valor com significância estatística. Fonte: Autores.

Quanto à forma com que os idosos com hipertensão arterial adquirem suas medicações (Tabela 3), aqueles que adquirem via SUS ou por doação possuem menor adesão à medicação, sendo esses resultados estatisticamente significativos $(\mathrm{p}=0,008$ e $\mathrm{p}<0,001$, respectivamente).

Tabela 3 - Distribuição das pessoas idosas com hipertensão arterial segundo forma com que adquire suas medicações e média de adesão à medicação, Rio Grande do Sul, Brasil, 2017.

\begin{tabular}{|c|c|c|c|c|}
\hline Variáveis & $\mathrm{n}(\%)$ & $\begin{array}{l}\text { Média de Adesão } \\
\text { à medicação e } \\
\text { desvio } \\
\text { padrão }(\bar{X} \pm D P)\end{array}$ & Valor $F / t$ & Valor $\mathrm{p}^{*}$ \\
\hline Adquire SUS & & & 2,665 & $0,008 * *$ \\
\hline Sim & $142(54)$ & $6,41 \pm 1,42$ & & \\
\hline Não & $122(46)$ & $6,65 \pm 1,72$ & & \\
\hline Adquire Farmácia & & & 1,828 & 0,072 \\
\hline Sim & $193(73)$ & $6,65 \pm 1,46$ & & \\
\hline Não & $71(27)$ & $6,15 \pm 1,78$ & & \\
\hline Adquire farmácia popular & & & 0,697 & 0,694 \\
\hline Sim & $179(68)$ & $6,61 \pm 1,51$ & & \\
\hline Não & $85(32)$ & $6,33 \pm 1,66$ & & \\
\hline Adquire doação & & & 3,979 & $<0,001 * *$ \\
\hline Sim & $3(1)$ & $5,33 \pm 3,05$ & & \\
\hline Não & 261(99) & $6,53 \pm 1,55$ & & \\
\hline
\end{tabular}

* Teste ANOVA. **Valor com significância estatística. Fonte: Autores. 


\section{Discussão}

A baixa taxa de adesão à medicamentos em hipertensos está associada a um maior risco de desenvolvimento de doenças cardiovasculares e hospitalizações. A prevalência de alta adesão do presente estudo foi de 36,7\%, já em estudo realizado com idosos de Juiz de Fora-MG a taxa de alta adesão foi de 47\% (Aquino et al, 2017). Por outro lado, quando esses valores são comparados com estudos realizados com a população adulta, a taxa de adesão é maior. Nesse sentido, estudo realizado com adultos, a partir de dados oriundos da Pesquisa Nacional sobre Acesso, Utilização e Promoção do Uso Racional de Medicamentos (PNAUM), a taxa de adesão nessa população foi de 69,2\% (Tavares et al, 2016).

A adesão ao tratamento exige uma grande responsabilidade do indivíduo sobre o seu tratamento, o que o torna um participante ativo nesse processo. Sendo o conhecimento que a pessoa possui sobre a sua doença um dos fatores mais importantes para integração e aceitação do regime terapêutico (Figueira et al, 2017), a presença de Diabetes ter se demonstrado relacionada a maiores médias de adesão à medicação pode estar associada a esse conhecimento prévio que o paciente que possui duas doenças crônicas ou mais pode apresentar.

Pessoas idosas com hipertensão arterial que faziam uso de medicamentos para o sistema digestivo/metabolismo obtiveram menores médias de adesão ao tratamento. Esse resultado pode estar relacionado a demanda de habilidades cognitivas e psicomotoras que medicamentos como a insulina requerem em sua autoadministração. É necessário que o usuário desses medicamentos compreenda aspectos que vão desde o seu armazenamento, transporte, preparo de solução e aplicação até o manuseio de seringas, agulhas ou canetas injetoras. Dessa forma, é necessário treinamento, mudanças no cotidiano e interesse em realizar o autocuidado, sendo essa situação mais preocupante nos idosos devido a fatores relacionados ao envelhecimento como déficit visual, cognitivo e motor, os quais dificultam a administração e podem comprometer a adesão ao tratamento (Vianna et al, 2017).

Aquelas pessoas idosas que adquiriam a medicação pelo SUS apresentaram menor adesão ao tratamento. Esse resultado pode estar relacionado à dificuldade de deslocamento até o local de entrega dos medicamentos que, embora possam ter sua oferta regular provida pelos gestores, o local disponibilizado, muitas vezes, é de difícil acesso aos idosos (Stefano et al, 2017). Porém, mesmo que no Brasil os pacientes tenham acesso gratuito ao Sistema Único de Saúde (SUS), um estudo que avaliou a disponibilidade dos medicamentos nas unidades de saúde pública no País encontrou baixa disponibilidade de medicamentos em todos os estratos populacionais (Mendes et al, 2014). Nesse sentido, acredita-se que esse fato deve ser avaliado e superado, visto que atrelado ao envelhecimento populacional, está o aumento da prevalência de doenças crônicas não transmissíveis, que demandam cuidados contínuos e utilização de medicamentos por tempo indeterminado. Facilitar o acesso a essas medicações, estabelecer fluxos, rotinas e diretrizes pode diminuir os custos, que tendem a ser cada vez mais representativos, e proporcionar maior qualidade no cuidado a essa população.

Ainda, adquirir a medicação por meio de doação também está relacionado a uma menor adesão ao tratamento. A falta de recursos financeiros para aquisição de medicamentos está entre os principais motivos relatados para a interrupção do tratamento (Remondi; Cabrera; Souza, 2014). Os usuários quando não encontram seus medicamentos no serviço público, não tem condições de compra-los ou não conseguem por meio de doação de parentes, vizinhos e amigos, deixam de utilizá-los. Dessa maneira, esse obstáculo pode inviabilizar o comportamento adequado para o controle da doença, favorecendo a suscetibilidade do usuário crônico a não adesão às medidas terapêuticas recomendadas (Becho; Almeida, 2017).

\section{Conclusão}

Dos idosos com hipertensão participantes do estudo, 36,7\% são aderentes e 63,3\% não aderentes. As variáveis que obtiveram relação estatisticamente significativa com as médias de adesão à medicação foram ter diabetes, fazer uso de medicamentos para o sistema digestivo/metabolismo e adquirir a medicação pelo SUS ou através de doação. 
O conhecimento das variáveis que estão relacionadas à adesão a medicação em pacientes idosos com hipertensão arterial contribui para o direcionamento das intervenções e das ações realizadas coordenadamente entre profissionais de saúde, pesquisadores, gestores e formuladores de políticas, a fim de melhorar o acesso e a adesão aos tratamentos, minimizando, consequentemente, agravos decorrentes dessa doença crônica.

Ainda, compreender detalhadamente as crenças, atitudes e motivações desses pacientes é essencial. São necessárias, portanto, novas pesquisas que avaliem qualitativamente a adesão a medicamentos de pessoas idosas com hipertensão arterial, com o intuito de determinar maior sucesso no tratamento dessa condição crônica e promover melhor qualidade de vida à essa população.

\section{Referências}

Abreu W. A., \& Portela N. L. C. R. (2015). Fatores associados à não adesão ao tratamento medicamentoso da Hipertensão Arterial Sistêmica. Interd. 8(3), 5060 .

Aquino G. A. et al. (2017). Fatores associados à adesão ao tratamento farmacológico em idosos que utilizam medicamentos anti-hipertensivo. Rev Bras Geriatr Gerontol, 20 (1), 116-27.

Baker D. W. et al. (1999). Development of a brief test to measure functional health literacy. Patient Educ Couns, 38(1), 33-42.

Barreto M. S., Carreira L., Marcon, S.S.(2015). Envelhecimento populacional e doenças crônicas: Reflexões sobre os desafios para o Sistema de Saúde Pública. Revista Kairós Gerontologia, 18(1), 325-39.

Becho A. S. et al.(2017). Dificuldades De Adesão Ao Tratamento Por Hipertensos De Uma Unidade De Atenção Primária À Saúde. Rev. APS. 20(.3), 349-59.

Bezerra A. L. A. et al.(2018). Perfil epidemiológico de idosos hipertensos no Brasil: uma revisão integrativa. Rev Med (São Paulo), 97(1), 103-7.

Brasil.(2010). Instituto Brasileiro de Geografia e Estatística. Cidades e Estados do Brasil. Municípios. Rio Grande RS. Pesquisas. Censo demográfico 2010: amostra - características da população; 2010. https://cidades.ibge.gov.br/brasil/rs/riogrande/pesquisa/23/25888?detalhes=true

Brasil. (2018). Ministério da Saúde. Secretaria de Vigilância em Saúde. Departamento de Vigilância de Doenças e Agravos não Transmissíveis e Promoção da Saúde. VIGITEL Brazil 2017: surveillance of risk and protective factors for chronic diseases by telephone survey: estimates of frequency and sociodemographic distribution of risk and protective factors for chronic diseases in the capitals of the 26 Brazilian states and the Federal District in 2017 Brasília; 2018.

Brasil (2007). Ministério da Saúde. Secretaria de Atenção à Saúde. Departamento de Atenção Básica. Envelhecimento e Saúde da Pessoa idosa. Ministério da Saúde, Secretaria de Atenção à Saúde. Departamento de Atenção Básica-Brasília: Ministério da Saúde, 2007. 192p.

Carthery-Goulart, M. T. et al. (2009). Performance of a Brazilian population on the test of functional health literacy in adults. Rev Saude Publica, 43(4), 63138 .

Duncan, B. B. et al.(2012). Doenças crônicas não transmissíveis no Brasil: prioridade para enfrentamento e investigação. Rev Saúde Pública, 46(1), 126-34.

Figueira, A. L. G. et al. (2017). Intervenções educativas para o conhecimento da doença, adesão ao tratamento e controle do diabetes mellitus. Rev Latino-Am. Enfermagem, 25(e2863).

Gil, A. C. (2017). Como elaborar projetos de pesquisa / Antonio Carlos Gil. (6a ed.), Atlas.

Girotto E. et al. (2013). Adesão ao tratamento farmacológico e não farmacológico e fatores associados na atenção primária da hipertensão arterial. Ciênc. saúde coletiva, 18(6), 1763-1772.

Mendes, L. V. et al. (2014). Disponibilidade de medicamentos nas unidades básicas de saúde e fatores relacionados: uma abordagem transversal. Saude Debate, 38, 109-23.

Morisky D.E. et al. (2008) Predictive Validity of A Medication Adherence Measure in an Outpatient Setting. J Clin Hypertens (Greenwich).

Moreira, R. M. et al. (2013). Qualidade de vida, saúde e política pública de idosos no Brasil: uma reflexão teórica. Revista Kairós Gerontologia, 16(1), 27-38, São Paulo.

Miott, H. A.(2011). Tamanho da amostra em estudos clínicos e experimentais. J Vasc Bras, 10(4), 275-8.

Oliveira-Filho, A. D. et al. (2012). Association between the 8- item Morisky Medication Adherence Scale (MMAS-8) and blood pressure control. Arq Bras Cardiol. 99(1), 649-58.

Organização Mundial da Saúde (2005). Envelhecimento ativo: uma política de saúde. Organização Pan-Americana de Saúde.

Remondi, F. A. et al.(2014). Não adesão ao tratamento medicamentoso contínuo: prevalência e determinantes em adultos de 40 anos e mais. Cad. Saúde Pública, 30(1), 126-36. 
Research, Society and Development, v. 10, n. 12, e343101220531, 2021

(CC BY 4.0) | ISSN 2525-3409 | DOI: http://dx.doi.org/10.33448/rsd-v10i12.20531

Santos, M. I. P. O., \& Portella, M. R. (2016). Conditions of functional health literacy of an elderly diabetics group. Rev Bras Enferm, 69(1), 144-52.

Sociedade Brasileira de Cardiologia (SBC). $7^{a}$ Diretriz Brasileira de Hipertensão Arterial. 107(3),

Stefano I. C. A. et al. (2017). Uso de medicamentos por idosos: análise da prescrição, dispensação e utilização num município de porte médio do estado de São Paulo. Rev. Bras. Geriatr. Gerontol. 20(5),681-92.

Tavares N. U. L. et al.(2016). Fatores associados à baixa adesão ao tratamento farmacológico de doenças crônicas no Brasil. Rev. Saúde Pública, 50(supl.2) São Paulo.

The PLOS Medicine Editors (2014) Observational Studies: Getting Clear about Transparency. PLoS Med, 11(8).

Vianna, M. S. et al. Competência para o autocuidado na administração de insulina por idosos septuagenários ou mais de idade. Rev Latino-Am. Enfermagem., 25(e2943).

World Health Organization (2003). Adherence to long term-therapies: evidence for action. Chapter 5, Towards the solution; 27-38. 